\title{
Evaluation of effects of chronic nasal steroid use on rhinological symptoms of COVID-19 with SNOT-22 questionnaire
}

\author{
Akif İşlek $^{1}$ iD $\cdot$ Mustafa Koray Balcı ${ }^{2}$ D
}

Received: 9 November 2020 / Revised: 24 January 2021 / Accepted: 11 February 2021 / Published online: 24 February 2021

(c) Maj Institute of Pharmacology Polish Academy of Sciences 2021

\begin{abstract}
Background The benefits of corticosteroids for the treatment of COVID-19 infection are documented in the literature. The goal of the study is to compare the severity of rhinological symptoms of COVID-19 between patients with nasal steroid use (NSU) and the control group (CG) using the sino-nasal outcome test (SNOT-22) questionnaire.

Methods A face-to-face survey was conducted at a second referral state hospital between. Patients with a complete recovery from COVID-19 were included in NSU and CG groups. Two subscales of the SNOT-22 were filled by the patients. The frequency and duration of smell and taste loss and SNOT-22 scores were compared between the two groups.

Results Forty-seven patients were included in the study. Thirty-one patients were in CG and 16 patients in the NSU group. Twenty-four $(51.1 \%)$ patients were females and $23(48.9 \%)$ were males. The mean age was $41.4 \pm 8.6$ years. Olfactory dysfunction was detected in $12(75 \%)$ patients in the NSU group, and 31 (93.3\%) patients in the control group (CG). Gustatory dysfunction was seen in $10(62.5 \%)$ patients in the NSU group and $24(77.4 \%)$ patients NSU group. $(p=0.071,0.279$, respectively). The duration of the olfactory $(6.6 \pm 2.5$ days $)$ and gustatory dysfunction $(6.1 \pm 2.6$ days $)$ and the mean SNOT-22 total score $(11.9 \pm 1.6)$ was significantly lower in the NSU group ( $p<0.001$, CI 11.1-5.1, CI 9.9-4.6, CI 9.3-5.9, respectively). Conclusions Although nasal steroid use does not prevent olfactory and gustatory dysfunction in COVID-19 patients, it may reduce the severity and duration of these symptoms.
\end{abstract}

Keywords COVID-19 $\cdot$ Nasal steroids $\cdot$ allergic rhinitis $\cdot$ SNOT-22 $\cdot$ olfactory dysfunction $\cdot$ Gustatory dysfunction

\section{Introduction}

The coronavirus disease 2019 (COVID-19) is caused by severe acute respiratory syndrome coronavirus 2 (SARS$\mathrm{CoV}-2$ ) and is so far responsible for more than 2 million deaths worldwide. The most prevalent symptoms are fever, cough, dyspnea, sputum production, myalgia, arthralgia, headache, diarrhea, rhinorrhea, sore throat, loss of olfactory and taste [1]. Olfactory and gustatory dysfunctions stand out as important diagnostic symptoms of the COVID19 infection which are detected in up to $90 \%$ of the cases [1]. Accompanying nasal symptoms are frequently present

Akif İşlek

drakifislek@gmail.com

1 Department of Otorhinolaryngology, Nusaybin State Hospital, Mardin, Turkey

2 Department of Otorhinolaryngology, İzmir Katip Celebi University Atatürk Training and Research Hospital, İzmir, Turkey especially in the early stages of the disease [2]. The SinoNasal Outcome Test-22 (SNOT-22) is a widely accepted questionnaire for objective measurement of rhinological symptoms of sino-nasal diseases [3]. Also, this questionnaire has been divided into four validated subscales representing categorical symptoms: nasal, otologic/facial, sleep-related, and emotional symptoms. The validity and reliability of SNOT-22 in the Turkish language have been previously confirmed [4].

Nasal steroids are frequently used for the treatment of allergic or non-allergic rhinitis and chronic sinusitis [5, 6]. They decrease the recruitment of inflammatory cells to the airway mucosa, selectively suppress local cytokine expression, inhibit mediator release, and support normal mucosal structure [7]. Minshall et al. [7] showed the decrease of focal metaplasia within the nasal epithelium after long-term use of mometasone by biopsy specimens. Recent studies suggest that nasal steroid treatments should be continued in patients with allergic rhinitis during COVID-19 pandemic [8]. Besides, stopping oral or inhaled corticosteroid treatments 
are not recommended due to the potentially dangerous worsening of asthma or allergic airway symptoms [8]. The use of nasal corticosteroids for COVID-19-related anosmia or ageusia is controversial. In the literatüre, there is a lack of evidence-based data regarding this topic.

SARS-CoV-2 uses the SARS-CoV receptor ACE2 for adhesion and entry to the host cell. Recent studies revealed that ACE2 expression is higher at the nasal mucosa $[9,10]$. The ACE2 expression in human nasal epithelial cells is significantly suppressed with Dexamethasone, in vitro [10]. Post-viral olfactory dysfunction is a sensorineural disorder and the use of oral or intranasal steroids is a C-level recommendation for the treatment [11]. In the current study, we aimed to test the hypothesis: 'COVID-19 associated nasal symptoms will be milder in patients with chronic nasal steroid use'. SNOT-22 questionnaire was used for the assessment of nasal symptoms objectively. We also aimed to evaluate if nasal oral steroid treatment was beneficial for anosmia or ageusia arising from COVID-19 infection.

\section{Materials and methods}

The study was designed as a face-to-face survey at a second referral state hospital (Nusaybin State Hospital) between April 2020 and July 2020. Symptomatic patients with positive polymerase chain reaction $(\mathrm{PCR}+)$ for SARS-CoV-2 by nasopharyngeal swabs were included in the study after complete regression of the symptoms and a negative PCR (-) test. After obtaining verbal and written informed consent, the volunteers were queried and the SNOT-22 was completed by the patients. Two subscales of the questionnaire (nasal and otologic/facial pain, Q: 1-12, min. score:0, max. score: 60) were assessed. Patients were divided into two groups according to nasal steroid use (NSU). Patients who had NSU (intranasal mometasone furoate spray, $1 \times 200$ $\mathrm{mcg}$, once in a day) for the last six months for allergic rhinitis were included in the study group. Patients with a history of other regular systemic or topical treatment were excluded. In the control group, patients with a previous history of smell or taste dysfunction, sino-nasal surgery, psychiatric or neurological diseases, tobacco use, previous trauma, surgery and/or radiotherapy in head and neck region, pre-existing taste or smell dysfunctions, chronic rhinosinusitis with nasal polyposis, and patients younger than 18 years were excluded.

The demographic data of the patients were recorded. SNOT-22 scores were calculated and the duration of odor loss was determined. The results were compared between the NSU and the control group (CG) using the Mann-Whitney $U$ and Chi-squared $\left(\chi^{2}\right)$ test. Spearman Rho Correlation Coefficient for complaints of smell and taste loss with the score of odor and taste items in SNOT-22 was calculated. SPSS 22.0 program (IBM Corp., Armonk, NY, USA) was used for statistics. The study was carried out in accordance with the 1964 Helsinki Declaration and subsequent amendments. The institutional review board was approved by the local Provincial Health Directorate for the study (No: 37201737-2/4/2020).

\section{Results}

Forty-seven COVID-19 patients were included in the study. Sixteen patients were enrolled in the nasal steroid use (NSU) group and 31 patients in CG. Patients were 24 (51.1\%) females and 23 (48.9\%) males. The mean age was $41.4 \pm 8.6$ years. The most common complains were loss of smell $(n=41,87.2 \%)$, cough $(n=38,80.8 \%)$, loss of taste $(n=34,72.3 \%)$, and nasal obstruction $(n=27,57.4 \%)$, respectively. The olfactory and gustatory functions began to recover after an average of $11.9 \pm 6.1$ and $10.8 \pm 5.4$ days, respectively. The mean total SNOT-22 score was $16.9 \pm 4.5$ (min: 9, max: 25). Total SNOT-22 scores were significantly lower in the NSU group and the loss of smell and taste complaints showed earlier improvement in this group $(p<0.001$, Table 1).

The mean, standard deviation, and $\mathrm{p}$ values of the scores obtained from the questions in the SNOT-22 questionnaire according to the study groups are presented in Table 2. A significant positive correlation was detected between the smell and taste loss complaints and the relevant scale in SNOT-22 ( $p<0.001, r=609$ and $p=0.006, r=397)$ (Fig. 1$)$.

\section{Discussion}

Human $\mathrm{CoV}$ was formerly known as a rare cause of postviral olfactory dysfunction (PVOD) [12]. During the COVID-19 pandemic, up to $90 \%$ association with gustatory and olfactory dysfunction is reported as an indicative symptom of the virus infection $[1,13,14]$. In the current study, taste and smell dysfunctions were also among the main symptoms associated with COVID-19. Anamnesis could not be obtained regarding the initial symptoms of the patients, however, taste and smell complaints were the symptoms that recovered the latest. Therefore, these patients are often admitted to the otorhinolaryngology clinic after the regression of the disease.

The successful outcomes of systemic and topical steroid treatment for post-viral olfactory dysfunction (PVOD) are reported in the literature, but the level of evidence is low [11]. Sharif-Askari et al. reported downregulating ACE2 expression in the nasal tissues of patients with chronic rhinosinüsitis (CRS) after prolonged use of steroids [10]. The authors suggested the possible protective effect of steroids during these infections [10]. Nasal steroids are frequently 
Table 1 Distribution of the demographic characteristics and findings of the patients by study groups

\begin{tabular}{|c|c|c|c|c|c|c|}
\hline \multicolumn{2}{|l|}{$(n, \%)$} & \multicolumn{2}{|l|}{ CG } & \multicolumn{2}{|c|}{ NSU } & \multirow{2}{*}{$\begin{array}{ll}\begin{array}{l}p \\
\chi^{2}\end{array} & \\
& 0.609\end{array}$} \\
\hline Gender & Male & 16 & 51.6 & 7 & 43.8 & \\
\hline & Female & 15 & 48.4 & 9 & 56.3 & \\
\hline \multirow[t]{2}{*}{ OD } & - & 2 & 6.5 & 4 & 25.0 & 0.071 \\
\hline & + & 29 & 93.5 & 12 & 75.0 & \\
\hline \multirow[t]{2}{*}{ GD } & - & 7 & 22.6 & 6 & 37.5 & 0.279 \\
\hline & + & 24 & 77.4 & 10 & 62.5 & \\
\hline (Med, 75\%) & & Man & tney $U$ & & & \\
\hline Age & & 39 & 48 & 40 & 47 & 0.719 \\
\hline SNOT-22 total & & 20 & 22 & 12 & 13 & $<0.001$ \\
\hline Duration of OD & & 14 & 18 & 7 & 8.5 & $<0.001$ \\
\hline Duration of GD & & 13 & 15 & 6 & 8 & $<0.001$ \\
\hline
\end{tabular}

$N S U$ nasal steroid use, $C G$ control group, $O D$ olfactory dysfunctions, $G D$ gustatory dysfunctions
Table 2 The mean (m), standard deviation (SD), and $p$ values of the scores obtained from the SNOT-22 questionnaire

\begin{tabular}{lrrlllr}
\hline & CG & & & NSU & \multicolumn{2}{c}{$p^{*}$} \\
\cline { 2 - 3 } & $m$ & SD & & $m$ & SD & \\
\hline Need to blow nose & 1.5 & 0.7 & .6 & 0.5 & $<0.001$ \\
Nasal obstruction & 1.6 & 0.6 & 1.1 & 0.6 & 0.015 \\
Sneezing & 1.4 & 0.6 & .9 & 0.6 & 0.016 \\
Runny nose & 1.7 & 0.7 & 1.2 & 0.4 & 0.009 \\
Cough & 2.7 & 1.0 & 1.9 & 0.5 & 0.004 \\
Postnasal drip & 1.4 & 0.7 & 0.4 & 0.5 & $<0.001$ \\
Thick nasal discharge & 1.5 & 0.8 & 0.4 & 0.5 & $<0.001$ \\
Ear fullness & 0.5 & 0.5 & 0.6 & 0.5 & 0.613 \\
Dizziness & 0.8 & 0.4 & 0.8 & 0.4 & 0.764 \\
Ear pain & 0.2 & 0.4 & 0.1 & 0.3 & 0.341 \\
Facial pain and/or pressure & 2.2 & 1.0 & 0.9 & 0.3 & 0.011 \\
Loss of smell and taste & 4.2 & 1.0 & 3.1 & 1.3 & 0.001 \\
SNOT-22 Total & 19.6 & 3.1 & 11.9 & 1.7 & $<0.001$ \\
\hline
\end{tabular}

$N S U$ nasal steroid use, $C G$ control group

*Mann-Whitney $U$ test

used for the treatment of nasal inflammatory diseases such as allergic rhinitis or CRS $[5,15]$. In an experimental animal study, a decreased ACE2 expression is shown during eosinophilic and allergic airway inflammation [16]. Yamaya et al. [17] demonstrated that budesonide reduces rhinovirus count, replication, and concentrations of cytokines of primary cultures of human tracheal epithelial cells. Studies evaluating the positive effects of inhaled steroids and systemic dexamethasone treatment on COVID-19 are reported in the recent literature $[18,19]$. In the current study, our findings showed that SNOT-22 total scores were significantly lower and the loss of smell and taste complaints showed earlier improvement in the NSU group.
Ogimi et al. [20]. demonstrated a significant association between prolonged shedding of human coronavirus $(\mathrm{HCoV})$ with high-dose steroid use in patients with hematopoietic cell transplant. Waltl et al. [21] reported that betamethasone may decrease epithelial damage caused by rhinovirus by anti-inflammatory properties on immune cells. Kim et al. [22] showed the in vitro antiviral activity of budesonide via inhibiting the human rhinovirus replication by autophagy activation. The authors suggested budesonide as a therapeutic option. In addition, the duration and dose of NSU are not homogeneous among studies in the literature.

However, the number of patients in NSU group was insufficient to make a reliable conclusion regarding this topic. Besides, it would add value if nasal mucosal changes caused by chronic nasal steroid use was demonstrated histopathologically. Although the possible benefits of NSU are tried to be measured objectively with a questionnaire, the absence of an objective test for the chemosensory function is another important limitation of our study. In this study, NSU in the last 6 months was set as the inclusion criteria, but in practice, there are different treatment modalities such as 1 year for perennial rhinitis.

In accordance with the literature, we observed that the nasal steroids may provide milder rhinological symptoms and also a shorter recovery in COVID-19 patients. But the level of evidence of the current study is low to present any suggestions. Prospective and controlled studies with larger samples would help to provide more valid results regarding the effects of nasal steroids on the nasal mucosa during COVID-19 infection. Also, the immunohistochemical pathway of gustatory and olfactory dysfunction in COVID-19 patients is still not clearly understood. Nasal steroids may be used for the treatment of the above-mentioned complaints in these patients, but this approach cannot be presented as a general recommendation at this stage. 
Fig. 1 Average and total (TS) SNOT-22 scores in the nasal steroid use (NSU) and control group (CG)

\section{SNOT-22, Q: 1-12}

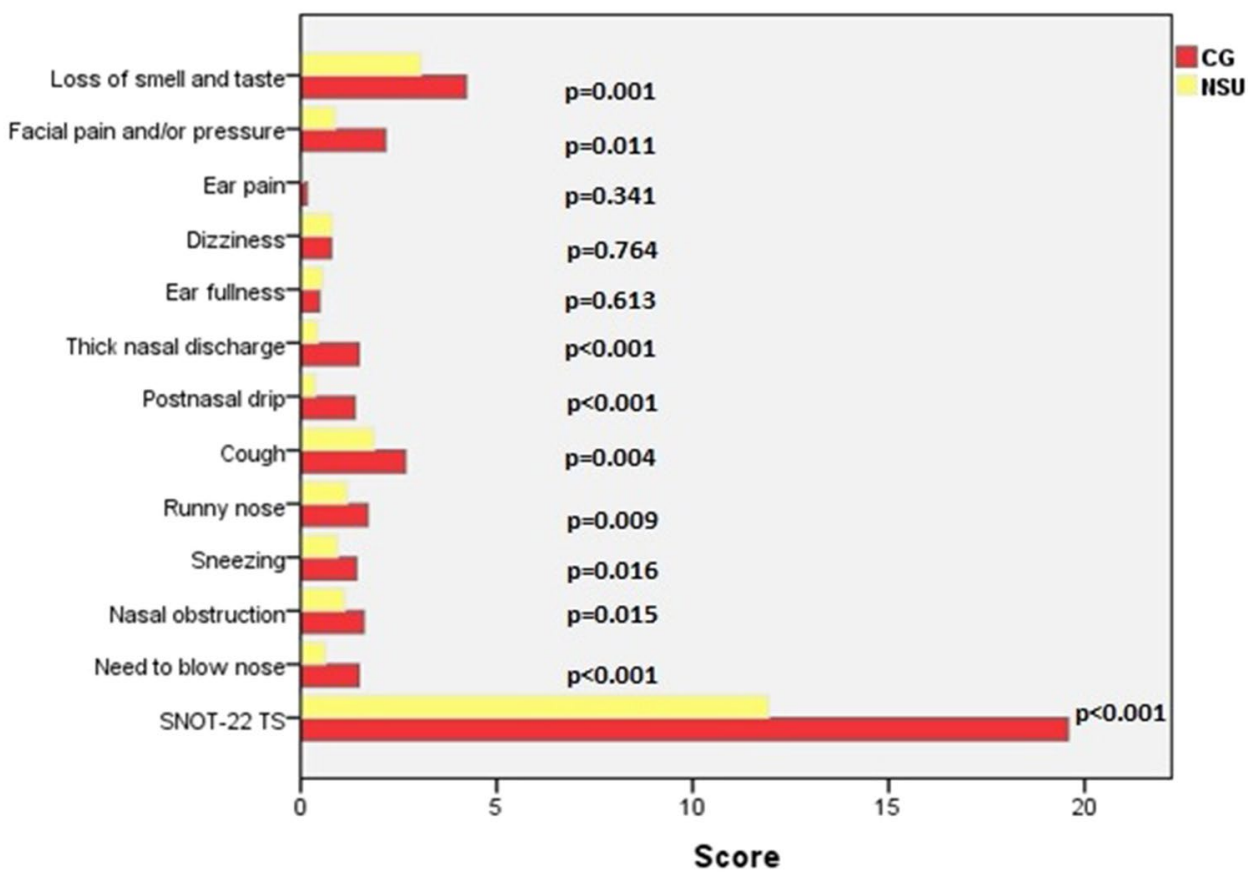

\section{Conclusion}

The use and benefits of corticosteroids in COVID-19 infection are still controversial. With the help of the SNOT-22 questionnaire, observed that patients with nasal steroid use had mild rhinological symptoms associated with COVID-19 infection. Although the level of evidence is low to come up with a suggestion, we observed that nasal steroids may be effective in the rapid recovery and improvement of olfactory and gustatory dysfunctions of these patients.

Funding This research did not receive any specific grant from funding agencies in the public, commercial, or not-for-profit sectors.

\section{Compliance with ethical standards}

Conflict of interest The authors declare no conflict of interest.

\section{References}

1. Lechien JR, Chiesa-Estomba CM, De Siati DR, Horoi M, Le Bon $\mathrm{SD}$, Rodriguez A, et al. Olfactory and gustatory dysfunctions as a clinical presentation of mild-to-moderate forms of the coronavirus disease (COVID-19): a multicenter European study. Eur Arch Otorhinolaryngol. 2020;277(8):2251-61. https://doi.org/10.1007/ s00405-020-05965-1.

2. Lechien JR, Cabaraux P, Chiesa-Estomba CM, Khalife M, Hans S, Calvo-Henriquez, et al. Objective olfactory evaluation of self-reported loss of smell in a case series of 86 COVID-19 patients. Head Neck. 2020;42(7):1583-90.

3. Morley AD, Sharp HR. A review of sinonasal outcome scoring systems: which is best? Clin Otolaryngol. 2006;31(4):103-9.

4. Cakir Cetin A, Kumus O, Keskinoglu P, Sutay S, Ecevit MC. Turkish validation of the Sino-nasal outcome test-22. Clin Otolaryngol. 2019;44(4):557-64.

5. Wise SK, Lin SY, Toskala E. International consensus statement on allergy and rhinology: allergic rhinitis-executive summary. Int Forum Allergy Rhinol. 2018;8(2):85-107.

6. Feng $\mathrm{CH}$, Miller MD, Simon RA. The united allergic airway: connections between allergic rhinitis, asthma, andchronic sinusitis. Am J Rhinol Allergy. 2012;26(3):187-90.

7. Minshall E, Ghaffar O, Cameron L, O'Brien F, Quinn H, RoweJones $\mathrm{J}$, et al. Assessment by nasal biopsy of long-term use of mometasone furoate aqueous nasal spray (Nasonex) in the treatment of perennial rhinitis. Otolaryngol - Head Neck Surg. 1998;118(5):648-54.

8. Bousquet J, Akdis C, Jutel M, Bachert C, Klimek L, Agache I, et al. Intranasal corticosteroids in allergic rhinitis in COVID-19 infected patients: an ARIA-EAACI statement. Allergy. 2020. https://doi.org/10.1111/all.14302.

9. Hoffmann M, Kleine-Weber H, Schroeder S, Krüger N, Herrler $\mathrm{T}$, Erichsen S, et al. SARS-CoV-2 cell entry depends on ACE2 and TMPRSS 2 and Is blocked by a clinically proven protease inhibitor. Cell. 2020;181(2):271-280.e8.

10. Saheb Sharif-Askari F, Saheb Sharif-Askari N, Goel S, Fakhri S, Al-Muhsen S, Hamid Q, et al. Are patients with chronic rhinosinusitis with nasal polyps at a decreased risk of COVID-19 infection? Int Forum Allergy Rhinol. 2020;10(10):1182-5. https ://doi.org/10.1002/alr.22672.

11. Hura N, Xie DX, Choby GW, Schlosser RJ, Orlov CP, Seal SM, et al. Treatment of post-viral olfactory dysfunction: an evidence-based review with recommendations. Int Forum Allergy Rhinol. 2020;10(9):1065-86. https://doi.org/10.1002/alr.22624. 
12. Suzuki M, Saito K, Min WP, Vladau C, Toida K, Itoh H, et al. Identification of viruses in patients with postviral olfactory dysfunction. Laryngoscope. 2007;117(2):272-7.

13. Wee LE, Chan YFZ, Teo NWY, Cherng BPZ, Thien SY, Wong $\mathrm{HM}$, et al. The role of self-reported olfactory and gustatory dysfunction as a screening criterion for suspected COVID-19. Eur Arch Otorhinolaryngol. 2020;277(8):2389-90. https://doi. org/10.1007/s00405-020-05999-5.

14. Martin-Sanz E, Riestra J, Yebra L, Larran A, Mancino F, YanesDiaz J, et al. Prospective study in 355 patients with suspected COVID-19 infection: value of cough, subjective hyposmia, and hypogeusia. Laryngoscope. 2020;130(11):2674-9. https://doi. org/10.1002/lary.28999.

15. Szefler SJ. Pharmacokinetics of intranasal corticosteroids. J Allergy Clin Immunol. 2001;108(1):26-31.

16. Dhawale VS, Amara VR, Karpe PA, Malek V, Patel D, Tikoo K. Activation of angiotensin-converting enzyme 2 (ACE2) attenuates allergic airway inflammation in rat asthma model. Toxicol Appl Pharmacol [Internet]. 2016;306:17-26. https://doi.org/10.1016/j. taap.2016.06.026.

17. Yamaya M, Nishimura H, Nadine L, Kubo H, Nagatomi R. Formoterol and budesonide inhibit rhinovirus infection and cytokine production in primary cultures of human tracheal epithelial cells. Respir Investig [Internet]. 2014;52(4):251-60. https://doi. org/10.1016/j.resinv.2014.03.004.

18. Halpin DMG, Singh D, Hadfield RM. Inhaled corticosteroids and COVID-19: a systematic review and clinical perspective.
Eur Respir J. 2020;55:5. https://doi.org/10.1183/13993003.01009 -2020 .

19. Johnson RM, Vinetz JM. Dexamethasone in the management of covid -19. BMJ. 2020;3(370):m2648. https://doi.org/10.1136/bmj. $\mathrm{m} 2648$.

20. Ogimi C, Greninger AL, Waghmare AA, Kuypers JM, Shean RC, $\mathrm{Xie} \mathrm{H}$, et al. Prolonged shedding of human coronavirus in hematopoietic cell transplant recipients: Risk factors and viral genome evolution. J Infect Dis. 2017;216(2):203-9.

21. Waltl EE, Selb R, Eckl-Dorna J, Mueller CA, Cabauatan CR, Eiwegger T, et al. Betamethasone prevents human rhinovirusand cigarette smoke- induced loss of respiratory epithelial barrier function. Sci Rep. 2018;8(1):1-10. https://doi.org/10.1038/s4159 8-018-27022-y.

22. Kim SR, Song JH, Ahn JH, Lee GS, Ahn H, Yoon SI, et al. Antiviral and anti-inflammatory activity of budesonide against human rhinovirus infection mediated via autophagy activation. Antiviral Res. 2018;151:87-96. https://doi.org/10.1016/j.antiv iral.2018.01.012.

Publisher's Note Springer Nature remains neutral with regard to jurisdictional claims in published maps and institutional affiliations. 